\title{
Inactivation of fission yeast Erh1 de-represses pho1 expression: evidence that Erh1 is a negative regulator of prt IncRNA termination
}

\author{
BEATE SCHWER, ${ }^{1}$ ANA M. SANCHEZ,${ }^{1,2}$ and STEWART SHUMAN ${ }^{3}$ \\ ${ }^{1}$ Department of Microbiology and Immunology, Weill Cornell Medical College, New York, New York 10065, USA \\ ${ }^{2}$ Gerstner Sloan Kettering Graduate School of Biomedical Sciences, New York, New York 10065, USA \\ ${ }^{3}$ Molecular Biology Program, Sloan Kettering Institute, New York, New York 10065, USA
}

\begin{abstract}
Fission yeast Erh1 exists in a complex with RNA-binding protein Mmi1. Deletion of erh1 up-regulates the phosphate homeostasis gene pho1, which is normally repressed by transcription in cis of a $5^{\prime}$ flanking prt lncRNA. Here we present evidence that de-repression of pho1 by erh1 $1 \Delta$ is achieved through precocious $3^{\prime}$-processing/termination of prt IncRNA synthesis, to wit: (i) erh1 $\Delta$ does not affect the activity of the prt or pho1 promoters per se; (ii) de-repression by erh1 $\Delta$ depends on CPF (cleavage and polyadenylation factor) subunits Ctf1, Dis2, Ssu72, Swd22, and Ppn1 and on termination factor Rhn1; (iii) de-repression requires synthesis by the Asp1 IPP kinase of inositol 1-pyrophosphates (1-IPPs); (iv) derepression is effaced by mutating Thr4 of the RNA polymerase II CTD to alanine; and (v) erh1 $1 \Delta$ exerts an additive effect on pho1 de-repression in combination with mutating CTD Ser7 to alanine and with deletion of the IPP pyrophosphatase Aps1. These findings point to Erh1 as an antagonist of IncRNA termination in the prt-pho1 axis. In contrast, in mmi1 cells there is a reduction in pho1 mRNA and increase in the formation of a prt-pho1 read-through transcript, consistent with Mmi1 being an agonist of prt termination. We envision that Erh1 acts as a brake on Mmi1's ability to promote CPF-dependent termination during prt IncRNA synthesis. Consistent with this idea, erh1 $1 \Delta$ de-repression of pho1 was eliminated by mutating the Mmi1-binding sites in the prt IncRNA.
\end{abstract}

Keywords: Erh1; Mmi1; Schizosaccharomyces pombe

\section{INTRODUCTION}

Fission yeast Erh1 is a 104-amino acid nuclear protein identified initially by virtue of its homology to the metazoan protein Enhancer of Rudimentary (Krzyzanowski et al. 2012). Schizosaccharomyces pombe erh $1 \Delta$ cells are viable but display abnormal morphology in stationary phase and sensitivity to several stressful growth conditions (Krzyzanowski et al. 2012). Subsequent studies implicated Erh1 in preventing the deleterious expression of meiotic genes during vegetative growth, which it accomplishes via its physical association with Mmi1, a YTH-family RNA binding protein that recognizes DSR (determinant of selective removal) sequences in target RNAs and promotes their elimination by the nuclear exosome (Yamashita et al. 2013; Sugiyama et al. 2016; Shichino et al. 2018). Mmi1 and Erh1 colocalize in nuclear foci in vegetative cells and are recovered as a stoichiometric Mmi1-Erh1 complex after Erh1-af-

Corresponding authors: bschwer@med.cornell.edu, s-shuman@ ski.mskcc.org

Article is online at http://www.rnajournal.org/cgi/doi/10.1261/rna. 076463.120 . finity purification from fission yeast cell extracts (Sugiyama et al. 2016). Mmi1 consists of: (i) a carboxy-terminal YTH-fold RNA binding domain with a distinctive mode of recognition of the DSR element UNAAAC and (ii) a lowcomplexity unstructured amino-terminal domain that is needed for formation of nuclear foci and mediates binding to Erh1 (Chatterjee et al. 2016; Wang et al. 2016; Shichino et al. 2018; Stowell et al. 2018; Xie et al. 2019). A crystal structure of Erh1 (expressed as a fusion in cis to a 28-aa segment of Mmi1 that suffices for binding to Erh1) showed that Erh1 is a homodimer and that each protomer has a Mmi1 peptide bound on its surface (Xie et al. 2019). In addition to its role in targeting RNA decay, Erh1 has imputed functions in the assembly of facultative heterochromatin (Sugiyama et al. 2016; Xie et al. 2019).

(C) 2020 Schwer et al. This article is distributed exclusively by the RNA Society for the first 12 months after the full-issue publication date (see http://rnajournal.cshlp.org/site/misc/terms.xhtml). After 12 months, it is available under a Creative Commons License (Attribution-NonCommercial 4.0 International), as described at http://creativecommons. org/licenses/by-nc/4.0/. 
Our interest in Erh1 was prompted by the results of transcription profiling of an erh $1 \Delta$ mutant whereby it was found that the phosphate homeostasis gene pho1 was up-regulated by sevenfold vis-à-vis a wild-type erh $1^{+}$control (Sugiyama et al. 2016). Fission yeast phosphate homeostasis entails a transcriptional response to phosphate availability that affects three genes that comprise a $\mathrm{PHO}$ regulon encoding proteins involved in extracellular phosphate acquisition, these being a cell surface acid phosphatase Pho1, an inorganic phosphate transporter Pho84, and a glycerophosphate transporter Tgp1 (Carter-O'Connell et al. 2012). Expression of the pho1, pho84, and tgp1 genes is actively repressed during growth in phosphaterich medium by the transcription in cis of a long noncoding (Inc) RNA from the respective 5' flanking genes prt, prt2, and nc-tgp1 (Lee et al. 2013; Ard et al. 2014; Shah et al. 2014; Chatterjee et al. 2016; Garg et al. 2018a; Sanchez et al. 2018a).

Our studies have shown that the repressive action of the IncRNAs on the PHO genes is governed by the RNA polymerase II (Pol2) CTD code, the 3' cleavage/polyadenylation factor complex CPF, transcription termination factor Rhn1, and synthesis of inositol 1-pyrophosphates (1-IPPs) (Schwer et al. 2014, 2015; Chatterjee et al. 2016; Sanchez et al. 2018b, 2019). Current evidence supports a model for the repressive arm of phosphate homeostasis whereby transcription of the upstream IncRNA interferes with expression of the downstream mRNA genes by displacing the activating transcription factor Pho7 from its binding site(s) in the mRNA promoters that overlap the IncRNA transcription units (Carter-O'Connell et al. 2012; Schwer et al. 2017; Garg et al. 2018b, 2019). Genetic manipulations of the aforementioned governors can elicit either hyper-repression or de-repression of the PHO genes, as depicted in Figure 1. For example, a CTD-T4A mutation that precludes installation of the Pol2 $\mathrm{Thr}^{-}-\mathrm{PO}_{4}$ mark hyper-represses the $\mathrm{PHO}$ genes under phosphate-rich conditions, as do deletions or loss-of-function mutations in the Ctf1, Dis2, Ssu72, Swd22, and Ppn1 subunits of CPF, deletion of termination factor Rhn1, and ablation of the IPP kinase Asp1 that synthesizes 1,5-IP8 and 1-IP7 from 5-IP7 and IP6, respectively. In contrast, a CTD-S7A mutation that eliminates the Pol2 Ser7- $\mathrm{PO}_{4}$ mark de-represses the $\mathrm{PHO}$ genes in phosphate-replete cells, as do IPP pyrophosphatase-inactivating mutations that increase IP8. RNA analyses, transcriptomics, and genetic epistasis analyses cohere around a model whereby: (i) hyper-repression is the result of reduced IncRNA termination and hence increased transcription across the mRNA promoter; and (ii) de-repression results from precocious termination of IncRNA transcription prior to the mRNA promoter and thus relief from transcription interference.

In the present study, we find that ablating Erh1 de-represses pho1 expression in phosphate-replete cells via a pathway that depends on CTD-Thr4, CPF subunits Ctf1, Dis2, Ssu72, Swd22, and Ppn1, termination factor Rhn1, and synthesis of 1-IPPs. Deletion of erh1 exerts an additive effect on pho1 de-repression in combination with CTDS7A and deletion of the IPP pyrophosphatase Aps1. These genetic interactions, and the findings that erh1 $\Delta$ has no impact on the activity per se of the prt IncRNA and pho1 mRNA promoters, point to Erh1 as a negative influence on IncRNA termination in the prt-pho1 axis. In mmi1s cells there is a reduction in pho1 mRNA and increased production of a prt-pho1 read-through transcript, consistent with Mmi1 being a positive effector of IncRNA termination. We suggest that Erh1 acts as a damper on Mmi1 such that when Erh1 is absent Mmi1 is unrestrained in eliciting precocious prt termination and thus de-repression of pho1 mRNA synthesis.
A

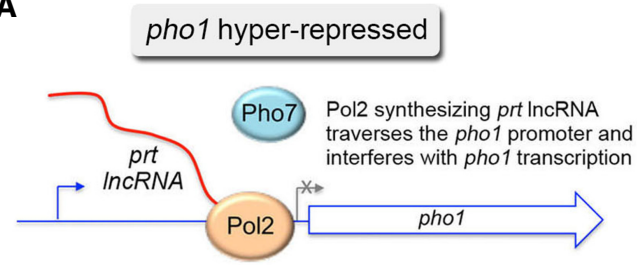

B

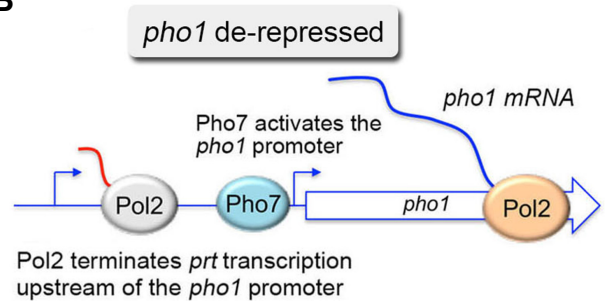

\section{CTD-T4A}

CPF mutants: ctf1 $\Delta$, dis $2 \Delta$, ssu72-C13S, ppn1 $1 \Delta$, swd22

$r h n 1 \Delta$ (termination factor)

asp1A; asp1-D333A (no IP8)
CTD-S7A

IPP pyrophosphatase mutants (high IP8)

erh1А

FIGURE 1. Synthesis of prt IncRNA represses pho1 mRNA transcription. Models for the pho1 hyper-repressed $(A)$ and de-repressed (B) states of the prt-pho1 locus under phosphate-replete conditions. The genetic perturbations that elicit these states are indicated at right. 


\section{RESULTS AND DISCUSSION}

\section{Deletion of Erh1 de-represses Pho1 expression}

We constructed a fission yeast erh $1 \Delta$ strain and tested the effects of Erh1 loss on growth on YES agar medium at $20^{\circ} \mathrm{C}$ to $37^{\circ} \mathrm{C}$ (Fig. 2A) and on pho1 expression during exponential growth at $30^{\circ} \mathrm{C}$ in liquid culture under phosphate-replete conditions (Fig. 2B). The erh1 $1 \Delta$ strain grew as well as the wild-type control strain at $30^{\circ} \mathrm{C}$ to $37^{\circ} \mathrm{C}$, as gauged by colony size, but displayed cold-sensitivity, manifest as small colony size at $25^{\circ} \mathrm{C}$ and failure to thrive at $20^{\circ} \mathrm{C}$ (Fig. 2A). Acid phosphatase activity (a gauge of Pho1 enzyme level that correlates with pho1 mRNA levels) was quantified by incubating suspensions of serial dilutions of the phosphate-replete cells for 5 min with p-nitrophenylphosphate and assaying colorimetrically the formation of p-nitrophenol. The basal Pho1 activity of wild-type cells was de-repressed by sixfold in erh1 $1 \Delta$ cells (Fig. 2B).

The loss of Erh1 might de-repress pho1 expression either by (i) reducing the activity of the prt IncRNA promoter that drives transcription interference with the pho1 promoter or (ii) increasing the intrinsic activity of the pho1 mRNA promoter independent of IncRNA synthesis. To address these issues, we used a plasmid-borne prt-pho1 reporter (Fig. 3A) that was introduced into fission yeast cells in which the chromosomal pho1 gene was deleted. This reporter faithfully reflects known homeostatic controls on the native pho1 locus (Chatterjee et al. 2016; Sanchez et al. 2019). We found that the prt-pho1 reporter was responsive to ablation of Erh1, whereby Pho1 levels under phosphate-replete conditions were de-repressed by eightfold in erh $1 \Delta$ cells compared to the wild-type erh $1^{+}$control (Fig. 3D). We then tested a mutated version of the prtpho1 reporter construct in which the prt promoter is inactivated by nucleotide changes in the HomolD and TATA box elements that drive prt IncRNA synthesis (Fig. 3B; Chatterjee et al. 2016). This mutant reporter provides a readout of the intrinsic activity of the pho1 promoter, absent interference by transcription of the flanking prt IncRNA. The Pho1 activity of the mutant plasmid in wildtype cells is high (i.e., de-repressed) and is not different from the Pho1 activity in erh $1 \Delta$ cells (Fig. 3E), thereby indicating that the de-repressive effect of erh $1 \Delta$ on pho1 expression from the wild-type prt-pho1 locus is not caused by up-regulation of the pho1 promoter per se. The effect of erh $1 \Delta$ on the prt promoter was assessed using a different plasmid reporter (Fig. $3 \mathrm{C}$ ) in which the prt promoter directly drives expression of the pho1 ORF. Pho1 expression from this plasmid was the same in wild-type and erh1 $\Delta$ cells (Fig. 3F), signifying that the de-repression of native pho 1 by erh $1 \Delta$ is not caused by decreased activity of the prt promoter. These results suggest that erh1 $1 \Delta$ affects pho 1 expression by enhancing precocious termination of prt IncRNA synthesis, à la the Pol2 rpb1-CTD-S7A allele (Fig. 1).
A
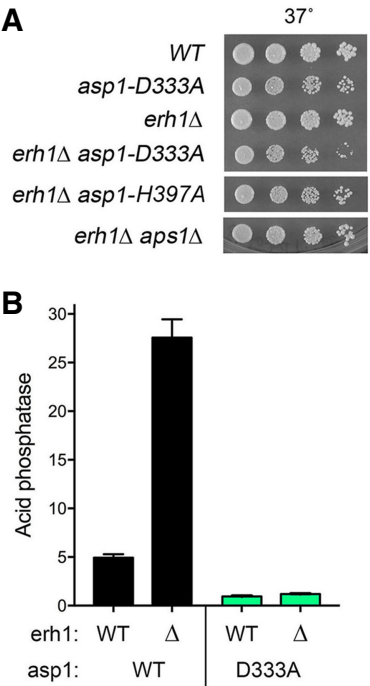
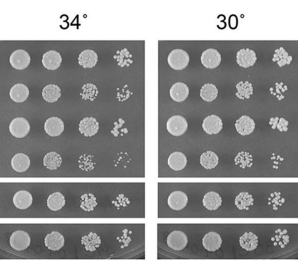

C

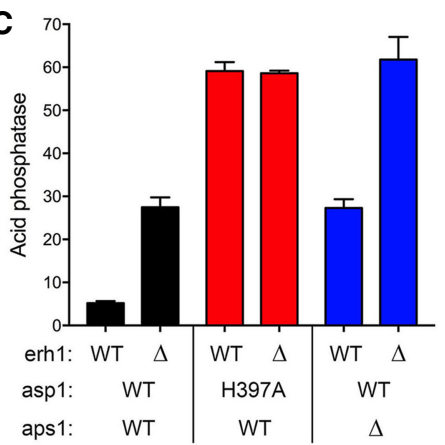

$25^{\circ}$

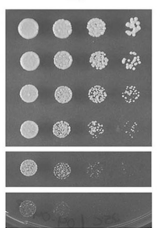

D

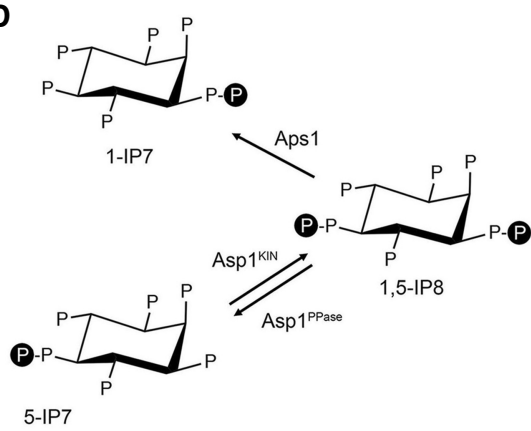

FIGURE 2. De-repression of Pho1 in erh1 $1 \Delta$ cells requires the synthesis of inositol 1-pyrophosphates by Asp1 kinase. (A) Growth of S. pombe strains with the indicated erh1, asp1, and aps1 alleles. Cells were inoculated in YES broth and grown at $30^{\circ} \mathrm{C}$. Exponentially growing cultures were adjusted to $A_{600}$ of 0.1 , and aliquots $(3 \mu \mathrm{L})$ of serial fivefold dilutions were spotted on YES agar and then incubated at the temperatures specified. $(B, C) S$. pombe strains with the indicated erh1, asp1, and aps1 alleles were grown to $A_{600}$ of 0.5 to 0.8 in liquid culture in YES medium at $30^{\circ} \mathrm{C}$. Cells were then harvested, washed with water, and assayed for Pho1 acid phosphatase activity by conversion of p-nitrophenylphosphate to $p$-nitrophenol. Activity is expressed as the ratio of $A_{410}$ ( $p$-nitrophenol production) to $A_{600}$ (input cells). Each datum in the bar graph is the average of assays using cells from at least three independent cultures \pm SEM. (D) Structures of the 5-IP7, 1-IP7, and 1,5-IP8 are shown. Asp1 kinase converts 5-IP7 to IP8 (and also IP6 to 1-IP7) and the Asp1 pyrophosphatase reverses this process. 
A

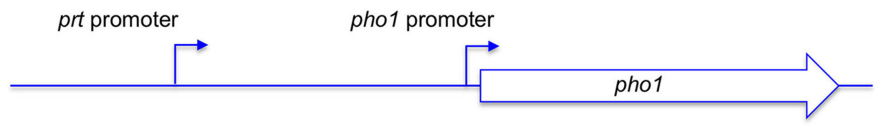

B

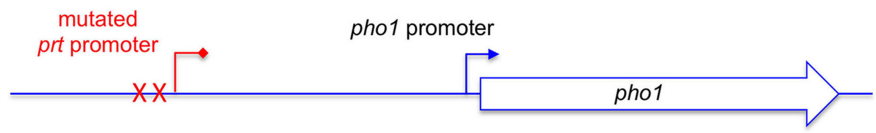

C prt promoter

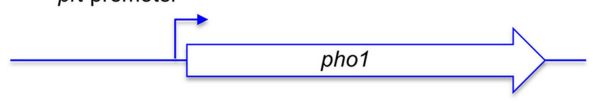

D

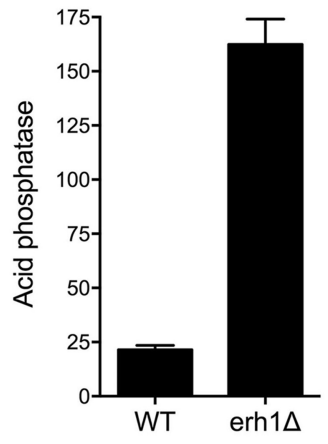

prt-pho1 reporter
E

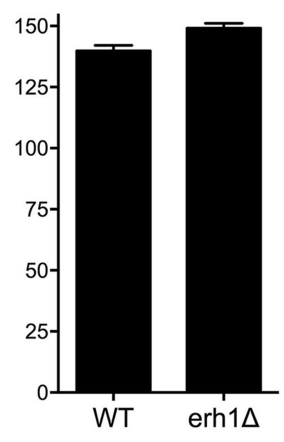

pho1 reporter
$\mathbf{F}$

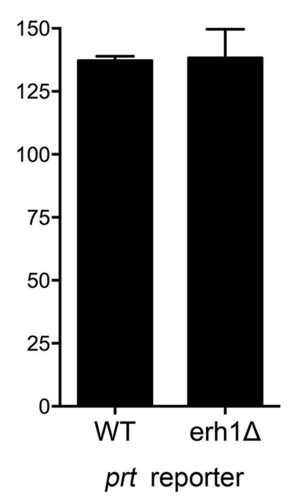

FIGURE 3. Does erh1 $1 \Delta$ affect the prt IncRNA or pho1 mRNA promoters? (A) Schematic of the plasmid-borne prt-pho1 reporter in which pho1 expression is repressed by prt IncRNA transcription. (B) A reporter of pho1 promoter activity in which prt IncRNA transcription is abolished by mutations (indicated by $\mathrm{X}$ ) in the HomolD and TATA box elements in the prt promoter (Chatterjee et al. 2016). (C) A reporter of prt promoter activity in which the prt promoter directly drives transcription of the pho1 gene. (D-F) The indicated reporter plasmids were transfected into $\operatorname{erh}^{+}$(WT) or erh1s strains in which the chromosomal pho1 locus was deleted. Transformants were selected and single colonies of individual transformants were pooled $(>20)$ and grown in plasmid-selective liquid medium to $A_{600}$ of $0.5-0.8$. Aliquots were harvested for acid phosphatase activity measurements. Each datum in the bar graph is the average of assays using cells from three independent cultures \pm SEM.

\section{erh1 $1 \Delta$ de-repression of Pho1 expression depends on 1-IPP synthesis}

Previous studies showed that de-repression of Pho1 elicited by the rpb1-CTD-S7A allele depends on the activity of the IPP kinase Asp1 (Sanchez et al. 2019). Asp1 is a bifunctional enzyme composed of an amino-terminal IPP kinase domain that converts 5-IP7 to 1,5-IP8, and a carboxy-terminal IPP pyrophosphatase domain that converts 1,5-IP8 back to 5-IP7 (Fig. 2D; Pascual-Ortiz et al. 2018; Dollins et al. 2020). Asp1 can also phosphorylate IP6 to yield 1-IP7 and de-phosphorylate 1-IP7 back to IP6. The in vivo effect of an asp1 $1 \Delta$ null allele or a kinase-dead asp1D333A allele is to eliminate intracellular IP8 and 1-IP7 and to increase the level of 5-IP7; the in vivo effect of a pyrophosphatase-defective asp1-H397A allele is to increase the level of IP8 (Pascual-Ortiz et al. 2018). To see if IPP status affects Pho1 de-repression by erh1 $\Delta$, we crossed erh $1 \Delta$ to an asp1-D333A strain. As noted previous- ly (Sanchez et al. 2019), asp1-D333A cells formed slightly smaller colonies than the wild-type control strain (Fig. 2A). The lack of IP8 in asp1-D333A cells resulted in a fivefold hyper-repression of Pho1 acid phosphatase activity (Fig. 2B). We obtained viable erh1s asp1-D333A haploids after mating and sporulation; the doublemutant was slow-growing on YES agar and cold-sensitive (Fig. 2A). The instructive finding was that the de-repression of Pho1 activity by erh $1 \Delta$ was erased in the asp1-D333A background (Fig. 2B), signifying a requirement for 1-IPP synthesis, presumably via 1-IPP's role as an agonist of 3'-processing/termination during prt transcription (Sanchez et al. 2019).

Pho1 is de-repressed in IP8 pyrophosphatase-defective asp1-H397A cells by virtue of precocious termination of prt IncRNA transcription when IP8 levels are elevated (Fig. 2C; Sanchez et al. 2019). Pho1 is also de-repressed in aps1 $1 \Delta$ cells (Fig. 2C; Sanchez et al. 2019) that lack the Nudix-family IPP pyrophosphatase Aps1, which converts 1,5-IP8 to 1IP7 (Fig. 2D; Safrany et al. 1999). Here we tested the genetic interactions of erh1 $1 \Delta$ with asp1-H397A and aps $1 \Delta$ by pairwise mating and recovery of viable erh1 $1 \Delta$ asp1-H397A and erh1 $\Delta$ aps1 $\Delta$ haploids after sporulation (Fig. 2A). The 11-fold de-repression of Pho1 activity elicited by asp1-H397A vis-à-vis wild-type asp $1^{+}$was virtually unaffected by the erh $1 \Delta$ deletion (Fig. 2C). In contrast, the fivefold de-repression of Pho1 in the aps1 $\Delta$ strain was enhanced additively to $12-$ fold in the erh1 $1 \Delta$ aps1 $1 \Delta$ background (Fig. 2C).

\section{De-repression of pho1 expression by erh1 $1 \Delta$ depends on CPF subunits and Rhn1}

To test our hypothesis that loss of Erh1 leads to precocious termination during prt IncRNA synthesis, we introduced the erh1s mutation into knockout strains lacking the Dis2, Ctf1, Ppn1, or Swd22 subunits of the CPF complex, a strain with a catalytically dead (C13S) version of the Ssu72 protein phosphatase subunit of CPF, and a strain that lacks the transcription termination factor Rhn1. Viable double-mutant haploids were recovered after pairwise matings and sporulation of the diploids (Fig. 4A). As noted previously (Sanchez et al. 2018b), the CPF and 
A

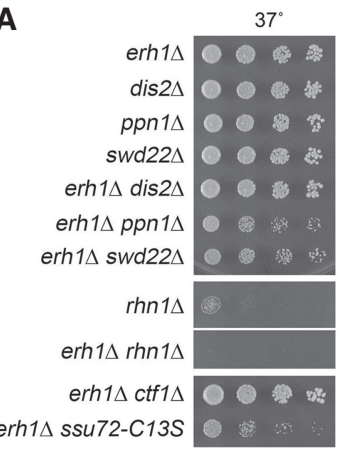

B

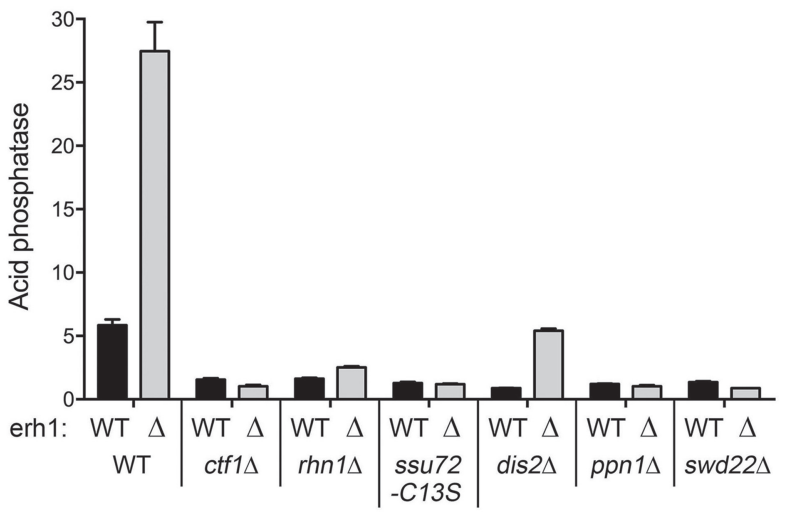

FIGURE 4. De-repression of pho1 expression by erh1 $1 \Delta$ depends on CPF subunits and Rhn1. (A) S. pombe strains with the genotypes indicated at left were inoculated in YES broth and grown at $30^{\circ} \mathrm{C}$. Exponentially growing cultures were adjusted to $A_{600}$ of 0.1 , and aliquots $(3 \mu \mathrm{L})$ of serial fivefold dilutions were spotted on YES agar and then incubated at the temperatures specified. (B) $S$. pombe strains bearing the indicated erh1 alleles (wild-type or erh1 $\Delta$ ) in combination with CPF subunit or Rhn1 mutations as specified were grown in liquid culture at $30^{\circ} \mathrm{C}$ and assayed for acid phosphatase activity.

Rhn1 mutations per se resulted in hyper-repression of Pho1 in phosphate-replete cells (Fig. 4B). The instructive findings were that the de-repression of Pho1 by erh1 $1 \Delta$

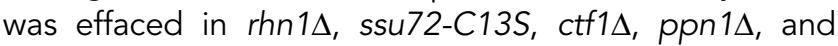
swd22 $\Delta$ cells and was attenuated in dis $2 \Delta$ cells (Fig. 4B). Thus, the increase in Pho1 expression in erh1 $\Delta$ cells requires CPF subunits and Rhn1, consistent with the precocious termination model (Fig. 1B).

\section{Genetic interactions of erh1s with Pol2 CTD phospho-site mutants}

The de-repressive effect of erh $1 \Delta$ on Pho1 expression and its genetic reliance on IP8 synthesis, CPF subunits, and Rhn1 reported above is similar to the IP8/CPF/Rhn1-dependent de-repression of pho1 observed in rpb1-CTDS7A cells and contrasts with the hyper-repression of Pho1 in rpb1-CTD-T4A cells (Chatterjee et al. 2016; Sanchez et al. 2018b, 2019). To query epistasis relationships, we mated erh $1 \Delta$ with the CTD phospho-site mutants and recovered viable haploid erh1 $1 \Delta$ CTD-S7A and erh1 $1 \Delta$
CTD-T4A progeny after sporulation (Fig. 5A). Assays of acid phosphatase activity showed that the S7A allele enhanced the de-repression of Pho1 by erh $1 \Delta$, whereas the T4A allele completely erased Pho1 de-repression (Fig. 5B). Thus, T4A "wins out" over erh1 $1 \Delta$ with respect to Pho1 expression, just as it does over other mutations (S7A, asp1-H397A, or aps1A) that de-repress the prtpho1 locus.

\section{Distinctive effects of erh $1 \Delta$ and mmi1s on pho1 expression}

Erh1 per se is a homodimer; it forms a 2:2 heterotetramer with Mmi1 (Sugiyama et al. 2016; Xie et al. 2019; Hazra et al. 2020). A pertinent question here is whether the pho1 de-repressive effects of erh $1 \Delta$ are recapitulated in the absence of Mmi1. To address this issue, we took advantage of the observation that Mmi1, which is essential for vegetative growth of fission yeast (by virtue of its ability to promote elimination of detrimental meiotic RNAs) can be deleted in a genetic background in which mei4 in inactivated, for example, in the mmi1s strain SP1111 (Sugiyama and Sugioka-Sugiyama 2011) where we find there is a Cys125-Stop nonsense mutation in the mei4 gene that truncates the 517-aa Mei4 protein. Figure 6A shows a northern blot analysis of total RNA from phosphate-replete wild-type, erh $1 \Delta$, and mmi1 $1 \Delta$ cells probed with a radiolabeled DNA oligonucleotide complementary to the pho1 ORF. Consistent with the Pho1 activity assays shown in the preceding sections, we find that the $\sim 1.6 \mathrm{~kb}$ pho1 mRNA is increased in erh1 $\Delta$ cells vis-à-vis wild-type. In contrast, the pho1 mRNA level is decreased slightly in
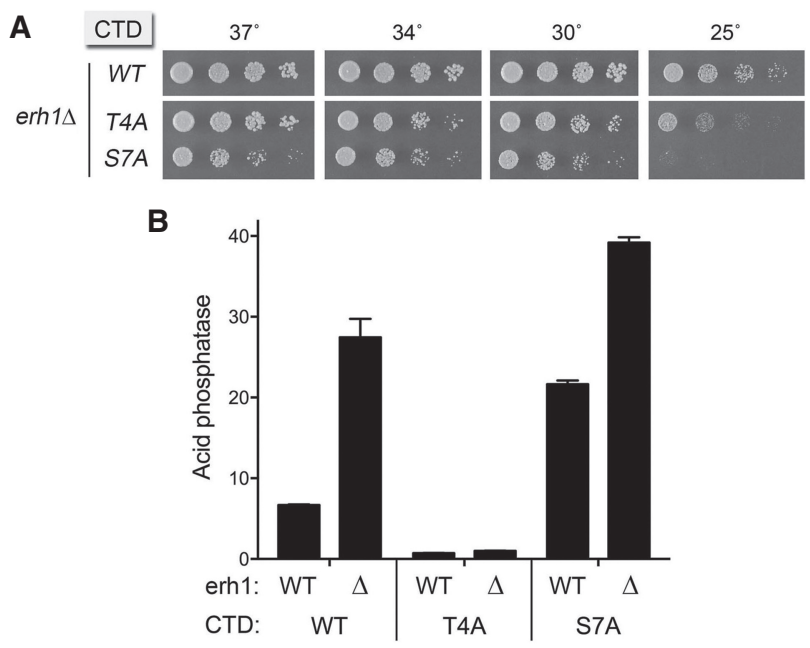

FIGURE 5. Genetic interactions of erh $1 \Delta$ with Pol2 CTD phospho-site mutants T4A and S7A. (A) Serial dilutions of $S$. pombe strains with the erh1 and rpb1-CTD alleles specified at left were spot-tested for growth at the indicated temperatures. (B) S. pombe strains with the indicated erh1 and rpb1-CTD alleles were grown in liquid culture at $30^{\circ} \mathrm{C}$ and assayed for acid phosphatase activity. 
A

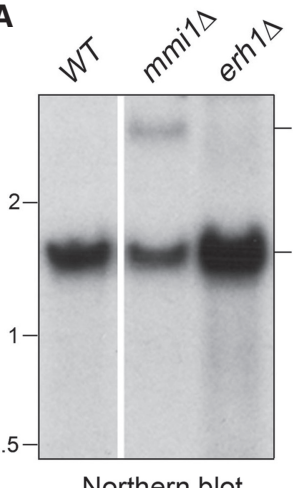

Northern blot

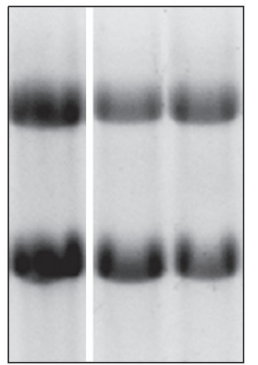

EtBr stain

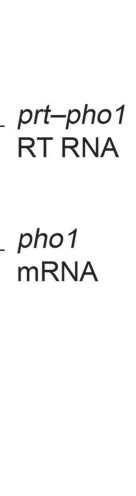

B
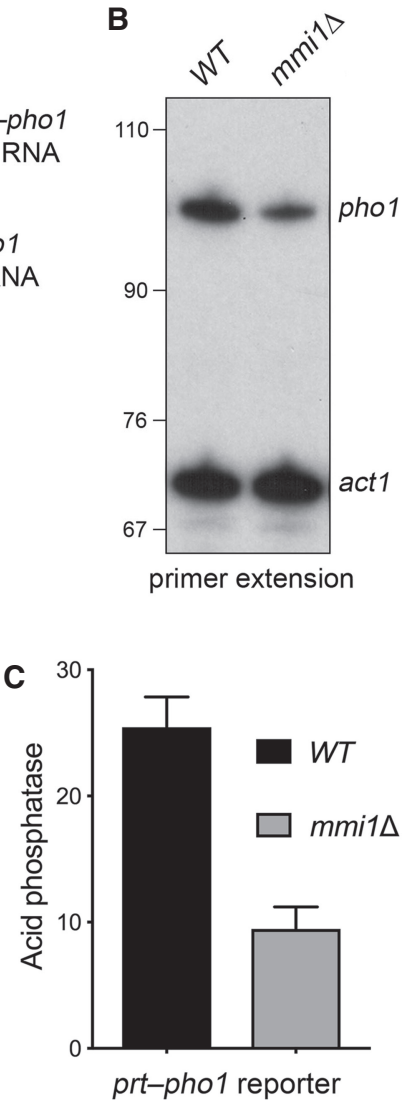

FIGURE 6. Distinctive effects of erh $1 \Delta$ and mmi1 $1 \Delta$ on pho 1 expression. (A) Northern analysis. RNAs isolated from WT, erh $1 \Delta$, and $\mathrm{mmi} 1 \Delta$ cells were resolved by formaldehyde-agarose gel electrophoresis and stained with ethidium bromide to visualize $28 \mathrm{~S}$ and $18 \mathrm{~S}$ ribosomal RNAs (bottom panel). The RNAs in the gel were transferred to membrane and hybridized to the pho1 probe. Annealed probe was visualized by autoradiography. The positions and sizes (in kilobases) of RNA markers are indicated on the left. The pho1 mRNA and prt-pho1 readthrough transcript are indicated on the right. The three RNA samples were analyzed in parallel on the same gel, probed on the same membrane, and visualized in a single exposure; intervening lanes of the stained gel and blot were cropped out during figure preparation. (B) Primer extension analysis. Total RNA from WT and mmi1 $1 \Delta$ cells was analyzed by reverse transcription primer extension using a mixture of radiolabeled primers complementary to the pho1 and act1 mRNAs. The reaction products were resolved by denaturing PAGE and visualized by autoradiography. The positions and sizes (nt) of DNA markers are indicated on the left. $(C) \mathrm{mmi}^{+}(\mathrm{WT})$ and $\mathrm{mmi} 1 \Delta$ cells deleted for pho1 and bearing the prt-pho1 reporter plasmid (shown in Fig. 3A) were assayed for acid phosphatase activity as described in Figure 3D.

mmi1 $\Delta$ cells and we detect the presence of a longer prtpho1 readthrough transcript (Fig. 6A) that extends from the prt IncRNA transcription start site to the pho1 mRNA poly(A) site. We also performed primer extension analysis to simultaneously gauge RNAs initiated from the transcription start sites of the pho1 and act1 protein-coding genes (Fig. 6B), which affirmed the presence of properly initiated pho1 mRNA in mmi1s cells at a slightly lower level than seen in wild-type cells. Our RNA analyses of mmi1 1 are in accord with RNA-seq data reported previously, whereby: (i) mmi $1 \Delta$ cells displayed increased read density over the prt IncRNA as well as persistent reads across the pho1 coding region (Kilchert et al. 2015; Xie et al. 2019); and (ii) erh $1 \Delta$ elicits a large increase in reads across the pho1 ORF but no apparent increase in reads for the prt IncRNA (Xie et al. 2019). We conclude that Erh1 and Mmi1 exert distinctive effects on pho1 expression at the RNA level. In addition, using the prt-pho1 reporter plasmid to gauge Pho1 acid phosphatase expression, we found that Pho1 activity was lower in mmi1s cells than in wild-type cells (Fig. 6C), an effect counter to the de-repression of Pho1 activity from the prt-pho1 reporter observed for erh $1 \Delta$ cells (Fig. 3D).

The data presented thus far suggest a scenario in which Erh1 acts as a brake on Mmi1's ability to promote CPF-dependent termination during prt IncRNA synthesis. Previous studies have shown that the steady-state level of Erh1 is unchanged in mmi1 $\Delta$ cells and the level of Mmi1 is similarly unaltered in erh1s cells (Sugiyama et al. 2016). Accordingly, we envision that when Erh1 is absent, Mmi1 is unrestrained in eliciting precocious prt termination and thus de-repression of pho1 mRNA synthesis. No such derepression is seen in mmi1s cells; rather there is a reduction in pho1 mRNA and increase in the formation of a prt-pho1 read-through transcript, reflective of decreased prt termination, augmented perhaps by decreased turnover of prt IncRNA in the absence of Mmi1. De-repression of pho1 by erh1 $1 \Delta$ depends on the CPF/Rhn1, CTD Thr4, and IP8/1-IP7 synthesis by Asp1. These are the same genetic dependencies that apply to pho1 de-repression triggered by CTD-S7A and by increased IP8 levels (in cells defective for IPP pyrophosphatase activity) (Sanchez et al. 2018b, 2019), the unifying theme being that such de-repression results from precocious prt termination upstream of the pho1 mRNA promoter (Fig. 1B). A nexus of Mmi1 in this interactome with respect to pho1 control is fortified by several recent observations from the Grewal laboratory: (i) Mmi1 is present in CPF complex affinity-purified from fission yeast; (ii) ChIP-seq data show that CPF and $\mathrm{Mmi1}$ are present at the prt locus, prominently over the $5^{\prime}$ half of the IncRNA (wherein reside the sites to which Mmi1 binds); and (iii) the CPF ChIP signal over the prt locus is present but lower in mmi1 $\Delta$ cells (Vo et al. 2019). They propose that Mmi1 recruits CPF to promote 3 '-processing termination. Our model that Erh1 dampens Mmi1/CPF-dependent prt termination begets a prediction that the derepression of pho1 in erh1 $1 \Delta$ cells should depend on the binding sites for Mmi1 in the prt IncRNA.

\section{De-repression of pho1 by erh1 $1 \Delta$ depends on DSR elements in the prt IncRNA}

The prt IncRNA contains two clusters of DSR elementsDSR $\times 3-1$ and DSR $3-2$-each composed of three DSR 
hexanucleotide elements (Fig. 7). The DSR clusters in the nascent IncRNA are binding sites for Mmi1 (Kilchert et al. 2015; Chatterjee et al. 2016). Previous studies highlighted the role of the DSR clusters in prt-promoted pho1 repression, via tracking the effects of compound mutations (mut1 and mut2) introduced into each of the hexanucleotide motifs comprising the DSR $\times 3-1$ and DSR $\times 3-2$ sequences of the prt-pho1 reporter plasmid (Fig. 7; Chatterjee et al. 2016). As recapitulated here, mut1 and mut2 reduced acid phosphatase activity in phosphate-replete cells to $75 \%$ and $22 \%$ of the wild-type prt DSR control, respectively (Fig. 7). Combining the mut1 and mut2 DSR cluster mutations had an additive effect, reducing Pho1 activity to $11 \%$ of the wild-type prt level. The hyper-repressive effect of the prt DSR mut1+mut2 changes on Pho1 expression were shown previously to "win out" over the de-repressive effects of CTD-S7A and asp1-H397A (i.e., increased IP8), suggesting that DSRs are key to establish the precocious termination of prt IncRNA synthesis that underlies Pho1 de-repression (Chatterjee et al. 2016; Sanchez et al. 2019). Here we queried the effect of DSR mutations on

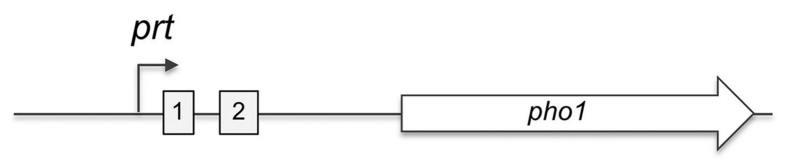

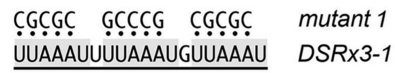

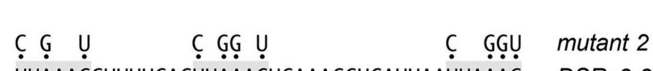

UUAAACCUUUUGAGUUAAACUGAAAGCUGAUUAAUUAAAC DSR $\times 3-2$

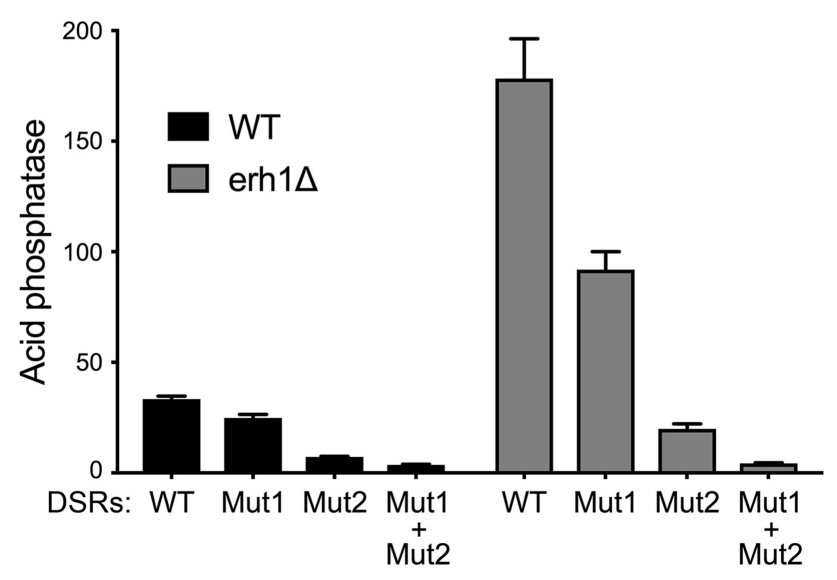

FIGURE 7. De-repression of pho1 by erh $1 \Delta$ depends on DSR elements in the prt IncRNA. The top panel shows a schematic of the prt-pho1 locus in the reporter plasmid that highlights the two clusters of triplet DSR elements recognized by Mmi1. The nucleotide sequence of the wild-type DSR clusters and the base mutations introduced into the mut1 and mut2 variants are shown below the graph. The reporter plasmids with wild-type or mutated DSRs were transfected into erh $1^{+}$(WT) or erh1 $1 \Delta$ strains in which the chromosomal pho1 locus was deleted. Acid phosphatase activity was determined as described in Figure 3D. de-repression of the prt-pho1 reporter in erh $1 \Delta$ pho1s cells. The mut1, mut2, and mut1+mut2 changes reduced Pho1 expression in erh $1 \Delta$ cells to $52 \%, 11 \%$, and $2 \%$, respectively, of the wild-type DSR reporter control, such that the level of Pho1 expression from the mut1+mut2 reporter was virtually identical in the wild-type and erh $1 \Delta$ strains (Fig. 7). We conclude that erh $1 \Delta$ de-repression of pho1 depends on the DSR elements in the prt IncRNA.

\section{Synthetic genetic interactions of mmi1 $\Delta$ with CPF and Rhn1 mutants}

There is significant functional redundancy built into the fission yeast $3^{\prime}$ processing/termination machinery, whereby the effects of ablating one inessential component are buffered by other inessential components, such that inactivation of both elicits synthetic lethality or severe synthetic sickness (Sanchez et al. 2018b, 2020). Because termination is one of several processes in which Mmi1 is implicated, we performed a synthetic genetic array analysis entailing pairwise mating of the mmi1s strain with ctf1 $1 \Delta$, ppn $1 \Delta$, swd22 $\Delta$, dis $2 \Delta$, ssu72-C13S, and $r h n 1 \Delta$ cells and screening large populations of random spores for the drug-resistance markers linked to the respective mutant alleles. We thereby

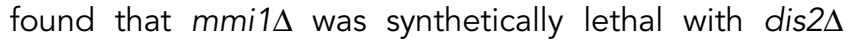
(Supplemental Fig. S1). The viable mmi1 $\mathrm{CPF} / \mathrm{rhn} 1$ strains were grown in YES liquid medium and then spot-tested for growth on YES agar in parallel with the mmi1s single mutant (which grew well at $25^{\circ} \mathrm{C}$ to $37^{\circ} \mathrm{C}$ ) (Supplemental Fig. S1). We found that: (i) $\mathrm{mmi} 1 \Delta$ was synthetically sick at all temperatures with ssu72-C13S, swd22 $\Delta$, and ppn $1 \Delta$; and (ii) mmi1s exacerbated the temperature sensitivity of rhn $1 \Delta$ such that mmi $1 \Delta$ rhn $1 \Delta$ cells failed to grow at $30^{\circ} \mathrm{C}$ (compare Supplemental Fig. S1 and Fig. 4A).

\section{Probing the structural requirements for Mmi1's essential function in vegetative cells}

The 488-aa Mmi1 protein (Fig. 8A) consists of carboxy-terminal YTH-fold RNA binding domain that suffices for DSR recognition (Chatterjee et al. 2016; Wang et al. 2016) and an unstructured amino-terminal domain that mediates protein-protein interactions. The Mmi1 segment from aa 95122 (underlined in Fig. 8A) suffices for interaction with Erh1 and forms an extensive hydrophobic and hydrogen-bonding interface with Erh1 in the cocrystal structure (Xie et al. 2019). The Mmi1 Erh1 complex interacts physically in vivo with the multisubunit Ccr4-Not deadenylase complex (Sugiyama et al. 2016), and Mmi1 (in the absence of Erh1) is reconstituted as a stoichiometric component of a highly purified recombinant $\mathrm{Mmi} 1 \bullet \mathrm{Ccr} 4-N o t$ complex produced by coexpression using a baculovirus vector (Stowell et al. 2016). Deletion of the amino-terminal 56-aa of Mmi1 abolished interaction with Ccr4-Not (Stowell et al. 2016). In order to interrogate the contributions of the amino- 
A

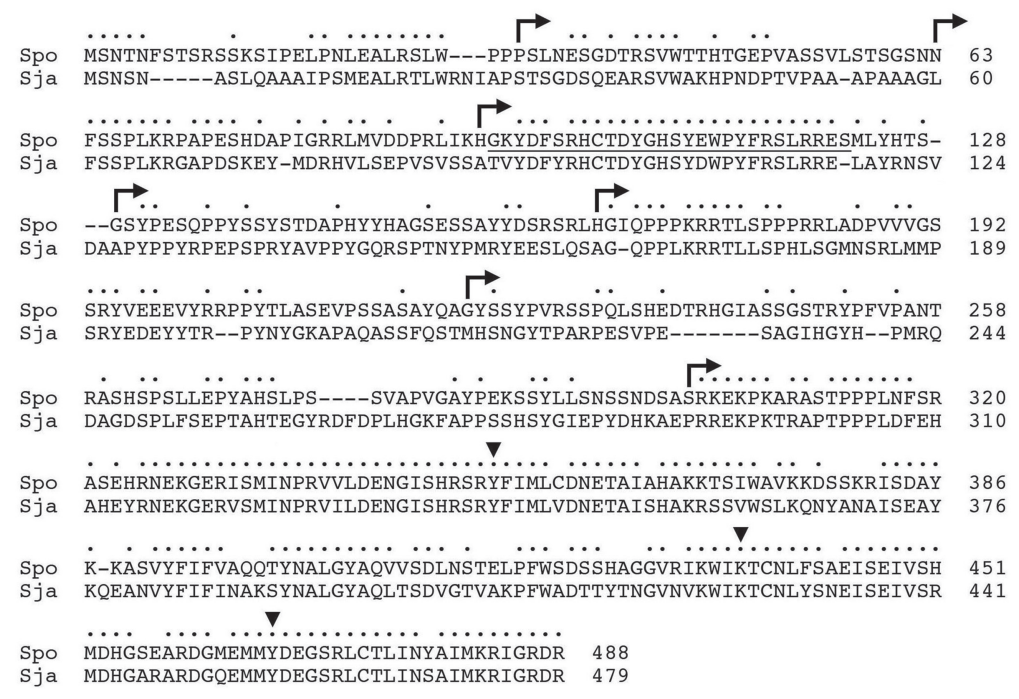

B
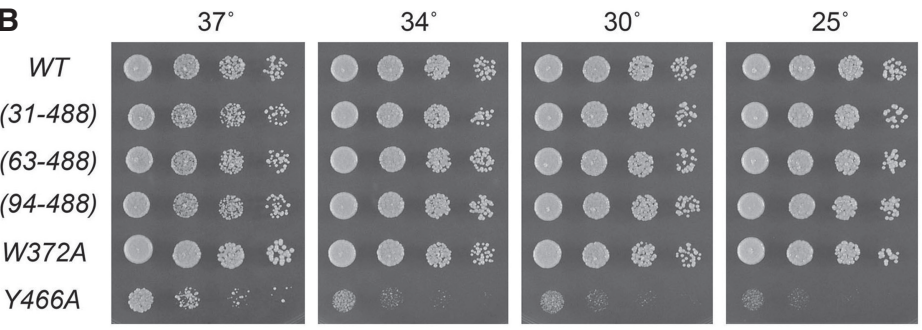

mmi1-(129-488), -(166-488), -(222-488) and -(301-488) are lethal mmi1-Y352A and $-K 436 A$ are lethal

FIGURE 8. Probing the structural requirements for Mmi1's essential function in vegetative cells. (A) The amino acid sequence of S. pombe Mmi1 (Spo) is aligned to that of the homologous protein from $\mathrm{S}$. japonicum (Sja). Positions of side chain identity/similarity are denoted by dots above the sequence. A conserved motif in the amino-terminal domain (aa 95-122) that suffices for Mmi1 interaction with Erh1 is underlined. The boundaries of the series of aminoterminal deletion alleles are indicated by rightward-pointing bent arrows. Amino acids in the carboxy-terminal YTH domain that were mutated to alanine are indicated by triangles above the sequence. (B) Viable mmi1- $\Delta N$ and mmi1-Ala strains were spot-tested for growth on YES agar at the indicated temperatures. Lethal mmi1- $\Delta N$ and mmi1-Ala alleles are indicated at bottom.

terminal region to Mmi1's essential function in vegetative cells, we introduced cDNAs for full-length mmi1 and a series of mmi1 amino-terminal truncation alleles, each marked by a $3^{\prime}$ flanking hyg $M X$ drug-resistance cassette, in lieu of one chromosomal $\mathrm{mmi}^{+}{ }^{+}$locus of a diploid fission yeast strain. The diploids were sporulated and large populations of random haploid progeny were screened for hygromycin resistance linked to the alleles of interest. We recovered viable haploid truncation mutants mmi1(31-488), mmi1-(63-488), and mmi1-(94-488) at 50\% frequency in the random spore analysis. Spot testing for growth on YES agar showed that these three truncation strains grew as well as the marked wild-type mmi1hygMX control strain (Fig. 8B). The inessential 93-aa amino-terminal segment includes the module required for Mmi1 binding to Ccr4-Not, suggesting that this proteinprotein interaction is dispensable for Mmi1 function during

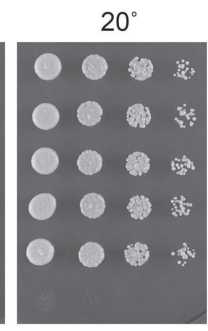

vegetative growth. In contrast, we were unable to recover any viable hygromycin-resistant haploids bearing the mmi1-(129-488), mmi1-(166488), mmi1-(222-488), or mmi1-(301488) alleles (Fig. 8). The loss of viability coincided with deletion of Mmi1 aa 94-128, which embraces the Erh1 interaction motif. Yet the lethality of mmi1-(129-488) is unlikely to reflect necessity for Erh1 $1 \mathrm{Mmi}^{1}$ interaction, insofar as (i) Erh1 per se is not essential and (ii) a single Mmi1 missense mutation W112A within the Erh1 binding motif that eliminates binding to Erh1 in vitro is able to support vegetative growth (Xie et al. 2019). It is conceivable that the Mmi1-(94-128) segment has a salutary effect on Mmi1 activity separate from its interaction with Erh1.

We also queried the role of RNA binding in Mmi1 vegetative function by alanine scanning of selected amino acids at the interface of the YTH domain with the DSR element $5^{\prime}-U^{1} U^{2} A^{3} A^{4} A^{5} C^{6}$ (Wang et al. 2016). We targeted RNA-binding residues Tyr352 (which makes hydrogen bonds to the $\mathrm{A}^{3}$ nucleobase), Lys436 (which makes hydrogen bonds to the $U^{1} \mathrm{pU}^{2}$ and $\mathrm{U}^{2} \mathrm{pA}^{3}$ phosphates), and Tyr466 (which makes a hydrogen bond to the $\mathrm{A}^{4}$ nucleobase). As a control, we mutated Trp372, a residue conserved among YTH proteins and a key component of the aromatic cage that recognizes the modified $\mathrm{m} 6 \mathrm{~A}$ nucleobase, but which is not involved in the binding of Mmi1 to DSR elements (Chatterjee et al. 2016; Wang et al. 2016). The salient findings were that alanine substitutions for DSR-binding residues Tyr352 and Lys436 were lethal and that a Y466A mutant was viable but extremely sick (Fig. 8B). In contrast, the aromatic cage mutant W372A grew as well as wild-type at $20^{\circ} \mathrm{C}-34^{\circ} \mathrm{C}$ and formed larger colonies than the WT control at $37^{\circ} \mathrm{C}$ (Fig. 8B). Thus, DSR recognition is essential for $\mathrm{Mmi1}$ to sustain vegetative growth.

\section{MATERIALS AND METHODS}

\section{Deletion of erh1}

Using PCR amplification and standard cloning methods, we constructed a plasmid in which the erh 1 gene from nucleotides +1 to 
+696 (relative to the translational start codon +1 ) was replaced by a kanMX antibiotic resistance cassette. The disruption cassette was excised from the plasmid and transfected into diploid $S$. pombe cells. G418-resistant transformants were selected and analyzed by Southern blotting to confirm correct integration at one of the erh 1 loci (thereby effectively deleting amino acids 1-99 of the 104-aa Erh1 protein). G418-resistant erh $1 \Delta$ haploids were isolated upon sporulation of the heterozygous diploids. A nourseothricin-resistant erh1 $\Delta$ strain was generated by marker switching (Hentges et al. 2005).

\section{Allelic exchange at the mmi1 locus}

Strains harboring marked wild-type and mutated mmi1 alleles were constructed as follows. We first built an integration cassette for wild-type mmi1 that consisted of five elements in series from $5^{\prime}$ to $3^{\prime}$ : (i) a 843-bp segment of genomic DNA $5^{\prime}$ of the mmi $1^{+}$start codon; (ii) a 1467-bp cDNA fragment encoding wild-type Mmi1, flanked by BamHI and HindIII restriction sites immediately adjacent to the translational start and stop codon, respectively; (iii) a 270-bp segment including poly(A)/termination signals from the $\mathrm{nmt}^{+}$gene; (iv) a hygMX gene conferring resistance to hygromycin; and ( $v$ ) a 815-bp segment of genomic DNA $3^{\prime}$ of the $\mathrm{mmi1}^{+}$stop codon. Amino-terminal truncation variants were generated by PCR using forward primers that introduced a BamHI site followed by a Met residue in lieu of Pro30, Asn62, Lys93, Ser128, Leu165, Ala221, or Ala300. Missense mutations in the full-length mmi1 ORF were introduced by two-stage PCR overlap extension with mutagenic primers. The mutated DNA restriction fragments were inserted into the integration cassette in lieu of the wild-type mmi1 ORF. All inserts were sequenced to exclude the presence of unwanted mutations. The integration cassettes were transfected into diploid S. pombe cells. Hygromycin-resistant transformants were selected and correct integrations at the target locus were confirmed by Southern blotting. The heterozygous diploids were then sporulated and hygromycin-resistant haploids were isolated. A segment of the mmi1-hygMX locus was amplified by $P C R$ and sequenced to verify that the desired mutations were present and to ascertain that the crossovers had occurred upstream of the translational start codon (i.e., that none of the introns were retained).

\section{Acid phosphatase activity}

Cells were grown at $30^{\circ} \mathrm{C}$ in YES liquid medium. Aliquots of exponentially growing cultures were harvested, washed with water, and resuspended in water. To quantify acid phosphatase activity, reaction mixtures $(200 \mu \mathrm{L})$ containing $100 \mathrm{mM}$ sodium acetate $(\mathrm{pH}$ 4.2), $10 \mathrm{mM}$ p-nitrophenyl phosphate, and cells (ranging from 0.01 to $0.1 A_{600}$ units) were incubated for $5 \mathrm{~min}$ at $30^{\circ} \mathrm{C}$. The reactions were quenched by addition of $1 \mathrm{~mL}$ of $1 \mathrm{M}$ sodium carbonate, the cells were removed by centrifugation, and the absorbance of the supernatant at $410 \mathrm{~nm}$ was measured. Acid phosphatase activity is expressed as the ratio of $A_{410}$ ( $p$-nitrophenol production) to $A_{600}$ (cells). The data shown in graphs are averages $( \pm$ SEM) of at least three assays using cells from three independent cultures.

\section{prt-pho1 reporter plasmids and assays}

The prt-pho1 reporter plasmids, marked with a kanamycin-resistance gene (kanMX), were transfected into pho1s cells (Chatterjee et al. 2016) or pho1 $1 \Delta$ erh $1 \Delta$ cells and transformants were selected on YES agar medium containing $150 \mu \mathrm{g} / \mathrm{mL}$ G418. Single colonies of individual transformants were pooled $(\geq 20)$ and grown in liquid YES + G418 medium to $A_{600}$ of $0.5-$ 0.8 . Aliquots were harvested by centrifugation for acid phosphatase activity measurements as described above.

\section{RNA analyses}

Total RNA was extracted via the hot phenol method (Herrick et al. 1990) from $20 A_{600}$ units of yeast cells that had been grown exponentially to $A_{600}$ of 0.6 to 0.8 at $30^{\circ} \mathrm{C}$. Aliquots $(15 \mu \mathrm{g})$ of total RNA were used as templates for M-MuLV reverse transcriptase-catalyzed extension of $5^{\prime}{ }^{32} \mathrm{P}$-labeled oligodeoxynucleotide primers complementary to the pho 1 or act 1 mRNAs. The primer extension reactions were performed as described previously (Schwer et al. 1998) and the products were analyzed by electrophoresis of the reaction mixtures through a $22-\mathrm{cm} 8 \%$ polyacrylamide gel containing $7 \mathrm{M}$ urea in $80 \mathrm{mM}$ Tris-borate, $1.2 \mathrm{mM}$ EDTA. The ${ }^{32} \mathrm{P}$-labeled primer extension products were visualized by autoradiography of the dried gel. The primer sequences were as follows: act1 5'-GATTTCTTCTTCCATGGTCTTGTC and pho1 5'GTTGGCACAAACGACGGCC. For northern blotting, aliquots $(10 \mu \mathrm{g})$ of total RNA were resolved by electrophoresis through a $1.2 \%$ agarose/formaldehyde gel. After photography under UV light to visualize ethidium bromide-stained rRNAs and tRNAs, the gel contents were transferred to a Hybond-XL membrane (GE Healthcare) and hybridization was performed with a randomly primed ${ }^{32} \mathrm{P}$-labeled DNA fragment spanning nucleotides 5901293 of the pho1 ORF.

\section{Mutational effects on fission yeast growth}

Cultures of $S$. pombe strains were grown in YES liquid medium until $A_{600}$ reached 0.6-0.8. The cultures were adjusted to $A_{600}$ of 0.1 and $3 \mu \mathrm{L}$ aliquots of serial fivefold dilutions were spotted on YES agar. The plates were photographed after incubation for $2 \mathrm{~d}$ at $34^{\circ} \mathrm{C}, 2.5 \mathrm{~d}$ at $30^{\circ} \mathrm{C}$ and $37^{\circ} \mathrm{C}, 4 \mathrm{~d}$ at $25^{\circ} \mathrm{C}, 6 \mathrm{~d}$ at $20^{\circ} \mathrm{C}$.

\section{Tests of mutational synergies}

Standard genetic methods were used to generate haploid strains harboring mutations/deletions in two differently marked genes. In brief, pairs of haploids with missense or null mutations were mixed on malt agar to allow mating and sporulation and then the mixture was subjected to random spore analysis. Spores $(\sim 1000)$ were plated on YES agar and on media selective for marked mutant alleles; the plates were incubated at $30^{\circ} \mathrm{C}$ for up to $5 \mathrm{~d}$ to allow slow growing progeny to germinate and form colonies. At least 500 viable progenies were screened by replicaplating for the presence of the second marker gene, or by sequentially replica-plating from YES to selective media. A finding that no haploids with both marker genes were recovered after 6 to $8 \mathrm{~d}$ of incubation at $30^{\circ} \mathrm{C}$ was taken to indicate synthetic lethality. By sequentially replica-plating and gauging the numbers 
of colonies at each step, we ensured that wild-type (unmarked) and the differentially marked single mutant alleles were recovered at the expected frequencies. Growth phenotypes of viable double-mutants were assessed in parallel with the individual mutants and wild-type cells at different temperatures $\left(20^{\circ} \mathrm{C}-37^{\circ} \mathrm{C}\right)$ as described above.

\section{SUPPLEMENTAL MATERIAL}

Supplemental material is available for this article.

\section{ACKNOWLEDGMENTS}

We thank Dr. Tomoyasu Sugiyama for generously providing the mmi1s strain SP1111. This work was supported by National Institutes of Health, National Institute of General Medical Sciences (NIH-NIGMS) grants R01-GM52470, R01-GM134021, and R35-GM126945.

Received May 16, 2020; accepted June 1, 2020.

\section{REFERENCES}

Ard R, Tong P, Allshire RC. 2014. Long non-coding RNA-mediate transcriptional interference of a permease gene confers drug tolerance in fission yeast. Nature Commun 5: 5576. doi:10.1038/ ncomms6576

Carter-O'Connell I, Peel MT, Wykoff DD, O'Shea EK. 2012. Genomewide characterization of the phosphate starvation response in Schizosaccharomyces pombe. BMC Genomics 13: 697. doi:10 .1186/1471-2164-13-697

Chatterjee D, Sanchez AM, Goldgur Y, Shuman S, Schwer B. 2016. Transcription of IncRNA prt, clustered prt RNA sites for Mmi1 binding, and RNA polymerase II CTD phospho-sites govern the repression of pho1 gene expression under phosphate-replete conditions in fission yeast. RNA 22: 1011-1025. doi:10.1261/rna.056515.116

Dollins DE, Bai W, Fridy PC, Otto JC, Neubauer JL, Gattis SG, Mehta KP, York JD. 2020. Vip1 is a kinase and pyrophosphatase switch that regulates inositol diphosphate signaling. Proc Natl Acad Sci 117: 9356-9364. doi:10.1073/pnas.1908875117

Garg A, Sanchez AM, Shuman S, Schwer B. 2018a. A long noncoding (Inc) RNA governs expression of the phosphate transporter Pho84 in fission yeast and has cascading effects on the flanking prt IncRNA and pho1 genes. J Biol Chem 293: 4456-4467. doi:10 .1074/jbc.RA117.001352

Garg A, Goldgur Y, Schwer B, Shuman S. 2018b. Distinctive structural basis for DNA recognition by the fission yeast $\mathrm{Zn}_{2} \mathrm{Cys}_{6}$ transcription factor Pho7 and its role in phosphate homeostasis. Nucleic Acids Res 46: 11262-11273. doi:10.1093/nar/gkx957

Garg A, Goldgur Y, Sanchez AM, Schwer B, Shuman S. 2019. Structure of fission yeast transcription factor Pho7 bound to pho1 promoter DNA and effect of Pho7 mutations on DNA binding and phosphate homeostasis. Mol Cell Biol 39: e00132-19. doi:10.1128/ MCB.00132-19

Hazra D, Andric V, Palancade B, Rougemaille M, Graille M. 2020. Formation of $S$. pombe Erh1 homodimer mediates gametogenic gene silencing and meiosis progression. Sci Rep 10: 1034. doi:10.1038/s41598-020-57872-4

Hentges P, Van Driessche B, Tafforeau L, Vandenhaute J, Carr AM. 2005. Three novel antibiotic marker cassettes for gene disruption and marker switching in Schizosaccharomyces pombe. Yeast 22: 1013-1019. doi:10.1002/yea.1291
Herrick D, Parker R, Jacobson A. 1990. Identification and comparison of stable and unstable RNAs in Saccharomyces cerevisiae. Mol Cell Biol 10: 2269-2284. doi:10.1128/MCB.10.5.2269

Kilchert C, Wittmann S, Passoni M, Shah S, Granneman S, Visiljeva L. 2015. Regulation of mRNA levels by decay-promoting intron that recruit the exosome specificity factor Mmi1. Cell Rep 13: 25042515. doi:10.1016/j.celrep.2015.11.026

Krzyzanowski MK, Kozlowska E, Kozlowski P. 2012. Identification and functional analysis of the erh $1^{+}$gene encoding enhancer of rudimentary homolog from the fission yeast Schizosaccharomyces pombe. PLoS One 7: e49059. doi:10.1371/journal.pone.0049059

Lee NN, Chalamcharia VR, Reyes-Turce F, Mehta S, Zofall M, Balachandran V, Dhakshnamoorthy J, Taneja N, Yamanaka S, Zhou M, et al. 2013. Mtr4-like protein coordinates nuclear RNA processing for heterochromatin assembly and for telomere maintenance. Cell 155: 1061-1074. doi:10.1016/j.cell.2013.10.027

Pascual-Ortiz M, Saiardi A, Walla E, Jakopec V, Künzel NA, Span I, Vangala A, Fleig U. 2018. Asp1 bifunctional activity modulates spindle function via controlling cellular inositol pyrophosphate levels in Schizosaccharomyces pombe. Mol Cell Biol 38: e00047-18. doi:10.1128/MCB.00047-18

Safrany ST, Ingram SW, Cartwright JL, Falck JR, McLennan AG, Barnes LD, Shears SB. 1999. The diadenosine hexaphosphate hydrolase from Schizosaccharomyces pombe and Saccharomyces cerevisiae are homologues of the human disphoshoinositol polyphosphate phosphohydrolase: overlapping substrate specificities in a MutT-type protein. J Biol Chem 274: 21735-21740. doi:10 .1074/jbc.274.31.21735

Sanchez AM, Shuman S, Schwer B. 2018a. Poly(A) site choice and Pol2 CTD Serine- 5 status govern IncRNA control of phosphate-responsive tgp1 gene expression in fission yeast. RNA 24: 237-250. doi:10.1261/rna.063966.117

Sanchez AM, Shuman S, Schwer B. 2018b. RNA polymerase II CTD interactome with $3^{\prime}$ processing and termination factors in fission yeast and its impact on phosphate homeostasis. Proc Natl Acad Sci 115: E10652-E10661. doi:10.1073/pnas.1810711115

Sanchez AM, Garg A, Shuman S, Schwer B. 2019. Inositol pyrophosphates impact phosphate homeostasis via modulation of RNA $3^{\prime}$ processing and transcription termination. Nucleic Acids Res 47: 8452-8469. doi:10.1093/nar/gkz567

Sanchez AM, Garg A, Shuman S, Schwer B. 2020. Genetic interactions and transcriptomics implicate fission yeast CTD prolyl isomerase Pin1 as an agent of RNA 3' processing and transcription termination that functions via its effects on CTD phosphatase Ssu72. Nucleic Acids Res 48: 4811-4826. doi:10.1093/nar/gkaa212

Schwer B, Mao X, Shuman S. 1998. Accelerated mRNA decay in conditional mutants of yeast mRNA capping enzyme. Nucleic Acids Res 26: 2050-2057. doi:10.1093/nar/26.9.2050

Schwer B, Bitton DA, Sanchez AM, Bähler J, Shuman S. 2014. Individual letters of the RNA polymerase II CTD code govern distinct gene expression programs in fission yeast. Proc Natl Acad Sci 111: 4185-4190. doi:10.1073/pnas.1321842111

Schwer B, Sanchez AM, Shuman S. 2015. RNA polymerase II CTD phospho-sites Ser5 and Ser7 govern phosphate homeostasis in fission yeast. RNA 21: 1770-1780. doi:10.1261/rna.052555.115

Schwer B, Sanchez AM, Garg A, Chatterjee D, Shuman S. 2017. Defining the DNA binding site recognized by the fission yeast $\mathrm{Zn}_{2} \mathrm{Cys}_{6}$ transcription factor Pho7 and its role in phosphate homeostasis. mBio 8: e01218-17. doi:10.1128/mBio.01218-17

Shah S, Wittmann S, Kilchert C, Vasiljeva L. 2014. IncRNA recruits RNAi and the exosome to dynamically regulate pho1 expression in response to phosphate levels in fission yeast. Genes Dev 28: 231-244. doi:10.1101/gad.230177.113

Shichino Y, Otsubo Y, Kimori Y, Yamamoto M, Yamashita A. 2018. YTH-RNA-binding protein prevents deleterious expression of 


\section{Schwer et al.}

meiotic proteins by tethering their mRNAs to nuclear foci. Elife 7: e32155. doi:10.7554/eLife.32155

Stowell JAW, Webster MW, Kögel A, Wolf J, Shelley KL, Passmore LA. 2016. Reconstitution of targeted deadenylation by the Ccr4-Not complex and the YTH domain protein Mmi1. Cell Rep 17: 19781989. doi:10.1016/j.celrep.2016.10.066

Stowell JAW, Wagstaff JL, Hill CH, Yu M, McLaughlin SH, Freund SMV, Passmore LA. 2018. A low-complexity region in the YTH domain protein Mmi1 enhances RNA binding. J Biol Chem 293: 9210 9222. doi:10.1074/jbc.RA118.002291

Sugiyama T, Sugioka-Sugiyama R. 2011. Red1 promotes the elimination of meiosis-specific mRNAs in vegetatively growing fission yeast. EMBO J 30: 1027-1039. doi:10.1038/emboj.2011.32

Sugiyama T, Thillainadesan G, Chalamcharla VR, Meng Z, Balachandran V, Dhakshnamoorthy J, Zhou M, Grewal SI. 2016. Enhancer of Rudimentary cooperates with conserved RNA-processing factors to promote meiotic mRNA decay and facultative heterochromatin assembly. Mol Cell 61: 747-759. doi:10.1016/j .molcel.2016.01.029
Vo TV, Dhakshnamoorthy J, Larkin M, Zofall M, Thillainadesan G, Balachandran V, Holla S, Wheeler D, Grewal SI. 2019. CPF recruitment to non-canonical transcription termination sites triggers heterochromatin assembly and gene silencing. Cell Rep 28: 267-281. doi:10.1016/j.celrep.2019.05.107

Wang C, Zhu Y, Bao H, Jiang Y, Xu C, Wu J, Shi Y. 2016. A novel RNA-binding mode of the YTH domain reveals the mechanism for recognition of determinant of selective removal by Mmi1. Nucleic Acids Res 44: 969-982. doi:10.1093/nar/ gkv1382

Xie G, Vo TV, Thillainadesan G, Holla S, Zhang B, Jiang Y, Lv M, Xu Z, Wang C, Balachandran V, et al. 2019. A conserved dimer interface connects ERH and YTH family proteins to promote gene silencing. Nature Commun 10: 251. doi:10.1038/s41467-01808273-9

Yamashita A, Takayama T, Iwata R, Yamamoto M. 2013. A novel factor Iss10 regulates $\mathrm{Mmi1}$-mediated selective elimination of meiotic transcripts. Nucleic Acids Res 41: 9680-9687. doi:10.1093/nar/ gkt763 

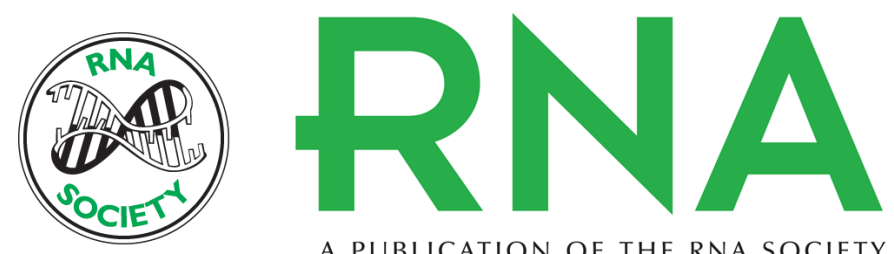

A PUBLICATION OF THE RNA SOCIETY

\section{Inactivation of fission yeast Erh1 de-represses pho1 expression: evidence that Erh1 is a negative regulator of prt IncRNA termination}

Beate Schwer, Ana M. Sanchez and Stewart Shuman

RNA 2020 26: 1334-1344 originally published online June 16, 2020

Access the most recent version at doi:10.1261/rna.076463.120

\section{Supplemental Material}

References

Creative Commons License

Email Alerting
Service
http://rnajournal.cshlp.org/content/suppl/2020/06/16/rna.076463.120.DC1

This article cites 33 articles, 15 of which can be accessed free at: http://rnajournal.cshlp.org/content/26/10/1334.full.html\#ref-list-1

This article is distributed exclusively by the RNA Society for the first 12 months after the full-issue publication date (see http://rnajournal.cshlp.org/site/misc/terms.xhtml). After 12 months, it is available under a Creative Commons License (Attribution-NonCommercial 4.0 International), as described at http://creativecommons.org/licenses/by-nc/4.0/.

Receive free email alerts when new articles cite this article - sign up in the box at the top right corner of the article or click here.

To subscribe to $R N A$ go to:

http://rnajournal.cshlp.org/subscriptions 